at baseline and in week 13. MRI and radiographic assessments of hand and wrist joints were performed at baseline and in week 26 .

The mean number of erosions detected by $\mathrm{MRI}$ at week 26 was significantly lower in the zoledronic-acid group than in the placebo group $(P=0.029)$. There was also a trend towards reduced development of new bone edema in patients in the zoledronic-acid group, compared with the placebo group. One limitation of this trial was the small number of patients; some of the observed differences between the groups did not reach statistical significance, which the authors attribute to the use of Wilcoxon's test.

The authors conclude that this trial confirms that osteoclasts are central to RA pathogenesis, and that zoledronic acid might be a useful addition to current RA therapy.

Original article Jarrett SJ et al. (2006) Preliminary evidence for a structural benefit of the new bisphosphonate zoledronic acid in early rheumatoid arthritis. Arthritis Rheum 54: 1410-1414

\section{TNF-inhibitor therapy safe and effective in spondyloarthritis}

The tumor necrosis factor (TNF) inhibitors infliximab, etanercept and adalimumab are commonly used in the treatment of both spondyloarthritis and rheumatoid arthritis (RA); however, longterm efficacy and safety data exist only for RA. A new study, based on data from the Spanish drug registry BIOBADASER, has compared the duration of treatment and safety profiles of these three TNF inhibitors in spondyloarthritis and rheumatoid arthritis.

Carmona and Gómez-Reino compared the rates of treatment discontinuation and adverse events in spondyloarthritis patients $(n=1,524)$ and RA patients $(n=4,006)$, respectively. At 1 , 2 and 3 years, they found that duration of treatment was markedly longer in spondyloarthritis than in RA. The hazard ratio for discontinuation of treatment among spondyloarthritis patients (compared with RA patients) was $0.66(95 \%$ $\mathrm{Cl}$ 0.57-0.76). In addition, adverse events were less common in spondyloarthritis patients: only $17 \%$ of spondyloarthritis patients experienced an adverse event, compared to $26 \%$ of RA patients $(P<0.001)$. The hazard ratio for adverse events in spondyloarthritis patients was 0.80 (95\% Cl 0.70-0.91).

The authors conclude that TNF-inhibitor therapy is safe and effective in spondyloarthritis; indeed, these agents have a better safety profile and longer duration of treatment when used to treat spondyloarthritis than when used to treat RA. As spondyloarthritis requires chronic treatment, however, assessment of outcomes beyond 3 years is recommended.

Original article Carmona L and Gómez-Reino JJ (2006) Survival of TNF antagonists in spondylarthritis is better than in rheumatoid arthritis. Data from the Spanish registry BIOBADASER. Arthritis Res Ther 8: R72

\section{Benefits of glucocorticoid therapy for RA are sustained after cessation}

Over the last 10 years, the debate over whether glucocorticoid therapy has joint-sparing and disease-modifying effects in rheumatoid arthritis (RA) has come to favor the existence of a beneficial effect. A Dutch team reported in 2002 that patients with early, active RA who were given $10 \mathrm{mg}$ prednisone daily for 2 years had less radiographically visible joint damage than patients who received placebo had; in their follow-up study, the team now reports that this beneficial effect of glucocorticoid therapy lasts for some years after prednisone is stopped.

The follow-up study was performed 3 years after the original, 2-year trial ended. The team evaluated 24 of the 40 patients who previously received prednisone and 28 of the 41 patients who previously received placebo. At the end of the first trial, prednisone therapy had been tapered over about 1 year and, therefore, had stopped $\sim 2$ years before the follow-up evaluation (in all but two patients, who continued to take prednisone).

In the follow-up study, patients who had previously taken prednisone still had less radiographically visible joint damage than those who had received placebo: the beneficial effect of prednisone was sustained. The authors claim that glucocorticoids could justifiably be considered to modify the course of early RA. Nonetheless, questions remain for future studies as to the optimal dosage and timing of glucocorticoid therapy. There could be a 'window of opportunity' during the early stages of RA within which glucocorticoids are most effective.

Original article Jacobs JWG et al. (2006) Followup radiographic data on patients with rheumatoid arthritis who participated in a two-year trial of prednisone therapy or placebo. Arthritis Rheum 54: 1422-1428 